\title{
A search for supernova remnants in the nearby spiral galaxy M74 (NGC 628)
}

\author{
E. Sonbaş ${ }^{1}$, A. Akyüz ${ }^{2}$, Ş. Balman ${ }^{3}$, and M. E. Özel ${ }^{4}$ \\ 1 University of Adiyaman, Department of Physics, 02040 Adiyaman, Turkey \\ e-mail: edasonbas@yahoo.com \\ 2 University of Çukurova, Department of Physics, 01330 Adana, Turkey \\ 3 Dept. of Physics, Middle East Technical University, 06531 Ankara, Turkey \\ ${ }^{4}$ Çağ University, Faculty of Arts and Sciences, 33800 Tarsus, Turkey \\ Received 12 December 2009 / Accepted 15 April 2010
}

\section{ABSTRACT}

\begin{abstract}
An optical search was carried out for supernova remnants (SNRs) in the Sc type nearby spiral galaxy M 74, using ground-based observations at the TUBITAK National Observatory (TUG, Antalya/Turkey) and the Special Astrophysics Observatory (SAO, Russia). Observations were supplemented by the spectral analysis of archived X-ray data from XMM-Newton and Chandra. The survey of M 74 covered $\sim 9 \operatorname{arcmin}^{2}$ with [S II], $\mathrm{H} \alpha$, and their continuum filters. Interference filter images of M 74 were obtained the with the $1.5 \mathrm{~m}$ Russian Turkish Telescope (RTT150) at TUG and spectral data taken with the $6 \mathrm{~m}$ Bolsoi Azimuthal Telescope (BTA) at SAO. The emission nebulae with continuum-subtracted line ratio values of [S II] $\lambda \lambda 6716,6731 / \mathrm{H} \alpha \geq 0.4$ are identified as SNRs. Followup spectroscopy confirmed optical SNR identifications. We have identified nine new SNR candidates in M 74 with [S II]/H $\alpha \geq 0.4$ as the basic criterion. The [S II]/H $\alpha$ ratio ranges from 0.40 to 0.91 and $\mathrm{H} \alpha$ intensities from $2.8 \times 10^{-15} \mathrm{erg} \mathrm{cm}^{-2} \mathrm{~s}^{-1}$ to $1.7 \times$ $10^{-14} \mathrm{erg} \mathrm{cm}^{-2} \mathrm{~s}^{-1}$. We also present spectral follow-up observations of these SNR candidates, however, we are able to spectrally confirm only three of them (SNR2, SNR3, and SNR5). The lack of confirmation for the rest might come from contamination by the nearby H II emission regions, as well as from the inaccurate positioning of the long slit on these objects. In addition, we searched the XMM-Newton and Chandra Observatory archival data for the X-ray counterparts to the optically identified candidates. We find positional coincidence with only three SNR candidates, SNR1, SNR2, and SNR8. The spectrum of SNR2 yields a shock temperature of $10.8 \mathrm{keV}$ with an ionization timescale of $1.6 \times 10^{10} \mathrm{~s} \mathrm{~cm}^{-3}$, indicating a relatively young remnant in an early Sedov phase, which is not supported by our optical wavelength analysis. Given the high luminosity of $10^{39} \mathrm{erg} \mathrm{s}^{-1}$ and the characteristics of the X-ray spectrum, we favor an ultra luminous X-ray source interpretation for this source associated with an SNR. We calculated an X-ray flux upper limit of $9.0 \times 10^{-15} \mathrm{erg} \mathrm{cm}^{-2} \mathrm{~s}^{-1}$ for the rest of the SNRs, including spectroscopically identified SNR3 and SNR5.
\end{abstract}

Key words. galaxies: groups: individual: M 74 (NGC 628) - ISM: supernova remnants - galaxies: ISM

\section{Introduction}

The brightest member of the small M 74 group in the Pisces constellation, M74 (NGC 628), is an Sc type spiral galaxy with a $\sim 6^{\circ}$ inclination angle at an assumed distance of $7.3 \mathrm{Mpc}$ (Sharina et al.1996; Soria et al. 2004). M 74 has well formed spiral arms with bright blue star clusters and dark cosmic dust lanes. In the early 2000s, two supernovae (SN 2002ap and SN 2003gd) were identified in M74 and studied extensively. The supernova event SN 2002ap is one of the four Type Ic SNe observed in X-rays (Van Dyk et al. 2003). SN 2003gd, which is found to be a nearby type II-P (plateau) event has a confirmed red giant progenitor (Hendry et al. 2005).

Leievre \& Roy (2000) have recovered well over 100 small and isolated H II regions and measured their fluxes in the extreme outer disk of M74 with $R>R_{25}$ making use of deep $\mathrm{H} \alpha$ narrowband imaging. They show that the massive star formation rate measured by the azimuthally averaged $\mathrm{H} \alpha$ surface brightness decreases monotonically from the center out to $R \sim 20 \mathrm{kpc}$, beyond which it drops rapidly.

Elmegreen et al. (2006) have examined the size and luminosity distributions of the star-forming regions in the galaxy with the Hubble Space Telescope. Their results suggest scale-free clumping of stars in the galaxy disk. Fathi et al. (2007) studied the internal kinematics of M74 over its entire face using FabryPerot interferometry with good angular resolution, and confirm the presence of an inner rapidly rotating disk-like component caused by the slow secular evolution of the large-scale spiral arms, together with the oval structure. They also detect over 300 $\mathrm{H}$ II regions in the galaxy with calibrated luminosities and diameters up to about $300 \mathrm{pc}$ using continuum subtracted narrow band images in $\mathrm{H} \alpha$.

M74 was observed in the X-rays using the Chandra and XMM-Newton data by Soria et al. (2004), and 74 discrete X-ray sources were detected in the combined Chandra observations to a detection limit of $\approx 6 \times 10^{36} \mathrm{erg} \mathrm{s}^{-1}$. They estimate that $15 \%$ of the M74 discrete sources have soft colors. They also calculated thermal SNRs with luminosities $\approx 2 \times 10^{37} \mathrm{erg} \mathrm{s}^{-1}$. Two bright X-ray sources (XMMU J013636.5+155036, CXOU $\mathrm{J} 13651.1+154547)$ were detected by XMM-Newton in M74 (Soria et al. 2004), while only the latter was detected by Chandra (Krauss et al. 2003). They established that both of the sources are variable. They also argue that there can be ultra luminous X-ray sources (ULX), but their true nature remains unclear.

Radio observations of M74 were carried out during the Arecibo Galaxy Environment Survey (AGES), which was designed to investigate M74 group environment in $21 \mathrm{~cm}$ with better sensitivity and higher spatial and velocity resolution than 
previous observations (Auld et al. 2006). They have obtained the spatial distribution of $\mathrm{HI}$ for M 74 and other selected galaxies.

In the UV regime, the surface brightness and color profiles for M 74 calculated by Cornett et al. (1994) show that the M 74 disk has sustained significant star formation over the last five hundred million years.

In this paper, we present our results for the search for new supernova remnant (SNR) candidates in M 74 using the observations with the RTT150 and 6 m-BTA telescopes. We also make use of archived Chandra data to look for their X-ray counterparts. These SNR studies are important for theories of interstellar medium (ISM) and star formation, since supernovae inject large amounts of matter and energy into the ISM. However, in spite of all the Galactic SNRs, their observations are impeded by several limitations such as the uncertainty on distances to individual objects and high extinctions along the line of sight in several regions of the Galactic plane. There are inherently fewer limitations and uncertainties in extragalactic samples. Assuming that all SNRs are the same distance from us for a given galaxy, we can easily compare their observed properties. The relative positions of such SNRs are determined with more precision. Once we know the positions of SNRs, their distances with respect to $\mathrm{H}$ II regions and spiral arms can be calculated easily (Matonick \& Fesen 1997; Blair \& Long 1997, 2004).

SNRs have been identified in a number of nearby spiral galaxies using optical observations (e.g. D'Odorico et al. 1980; Braun \& Walterbos 1993; Magnier et al. 1995; Matonick \& Fesen 1997; Matonick et al. 1997; Gordon et al. 1998, 1999; Blair \& Long 1997, 2004; Sonbas et al. 2009), X-ray observations (Pence et al. 2001; Ghavamian et al. 2005), and radio observations (Lacey et al. 1997; Lacey \& Duric 2001; Hyman et al. 2001). Multiwavelength surveys of SNRs have also been carried out by Pannuti et al. (2000, 2002, 2007).

In our work, we use the well known and accepted criterion ( $\mathrm{S} \mathrm{II} / \mathrm{H} \alpha \geq 0.4$ ) proposed by Mathewson \& Clarke (1973) to differentiate SNRs from typical H II regions. The rationale for this lies in the fact that, in a typical H II region, sulfur is usually expected to take the form of $\mathrm{S}^{++}$owing to strong photoionizing fluxes from central hot stars, making the ratio $[\mathrm{S} \mathrm{II}] / \mathrm{H} \alpha$, typically, in the range $\sim 0.1-0.3$. Shock waves produced by $\mathrm{SN}$ explosion propagate through the surrounding medium. The matter cools sufficiently behind these waves, and a variety of ionization states occur that include $\mathrm{S}^{+}$. This might be the reason for the increased $[\mathrm{S} \mathrm{II]} / \mathrm{H} \alpha$ ratio observed in SNRs. It follows that almost all discrete emission nebulae satisfying the above criterion are expected to be shock-heated.

The organization of the paper is as follows. In Sect. 2, we discuss our imaging and spectroscopic observations and related data reduction. Search results in the optical band, the identification of SNRs, and search for their X-ray counterparts are presented in Sect. 3. Finally, conclusions and discussions of our results are provided in Sect. 4.

\section{Observations and data reduction}

\subsection{Imaging}

The imaging observations of M74 were performed with the $1.5 \mathrm{~m}$ Russian Turkish Telescope (RTT150) at TUBITAK National Observatory (TUG) in Turkey. M 74 images were obtained using a Cassegrain imaging CCD Loral Lick $2 \mathrm{k} \times 2 \mathrm{k}$ back-illuminated and anti-reflection coated (single layer, $700 \AA$ of hafnium oxide, HfO2), $2048 \times 2048$ pixel CCD with a plate scale of 0.26 pixel $^{-1}$, giving a 9!1 × 9!1 field of view (FOV).

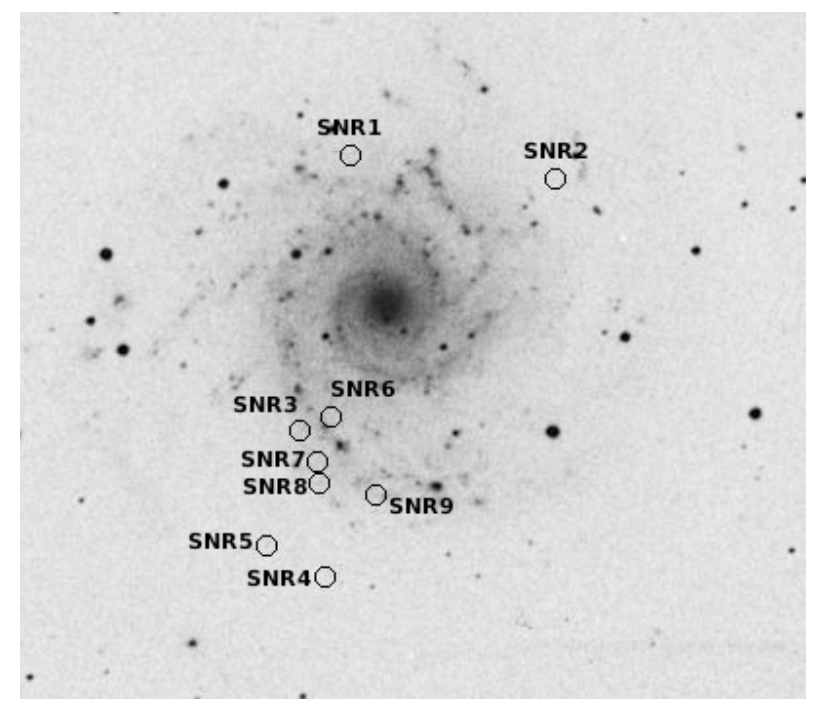

Fig. 1. Nine new SNR candidates found in this work are labeled with circles on the image of M 74 extracted from Digital Sky Survey (DSS).

Table 1. Characteristics of the interference filters used in our observations.

\begin{tabular}{ccc}
\hline \hline Name & $\lambda$ & $F W H M$ \\
& Wavelength $(\AA)$ & $\AA$ \\
\hline$[$ S II] & 6728 & 54 \\
continuum & 6964 & 350 \\
H $\alpha$ & 6563 & 80 \\
continuum & 6446 & 123 \\
\hline
\end{tabular}

We used narrowband interference filters centered on the lines of [S II], $\mathrm{H} \alpha$, and continuum filters to remove starlight from $\mathrm{H} \alpha$ and [S II] images. The interference filter characteristics used in these observations are shown in Table 1. Only one field image for each filter was obtained for M 74 on September 19 and 20, 2004. We have exposure times of $12 \times 300 \mathrm{~s}$ for the [S II], $\mathrm{H} \alpha$ filters, and $4 \times 300$ s for the two continuum filters. This way, we were able to obtain deeper field images with a higher signal-to-noise ratio for the faintest objects.

The European Southern Observatory Munich Image Data Analysis System (ESO-MIDAS) software environment (version 08FEBpl1.1) was used to reduce the data (http://www.eso. org). We observed two or three standard stars each night from the list of Oke (1974) and Stone (1977) to determine the average flux conversion factors (FCF). We used the technique from Jacoby et al. (1987) to calculate this factor in the units of erg $\mathrm{cm}^{-2} \mathrm{~s}^{-1} / \mathrm{ADU} \mathrm{s} \mathrm{s}^{-1}$. FCF allows us to convert measured counts from an image to a flux in units of $\mathrm{erg} \mathrm{cm}^{-2} \mathrm{~s}^{-1}$. Bias and flat-field frames for each image were also obtained. This way each exposure was bias-subtracted, trimmed, and flat-fielded. The cosmic hits were also removed from each [S II] and $\mathrm{H} \alpha$ image. Astrometry was applied to the resulting [S II] and $\mathrm{H} \alpha$ images to include world coordinate system information into the FITS header of the individual images of M 74. Red stars from USNO A2.0 catalog (Monet et al. 1998) were used to check positions of the stars. In Fig. 1, we show the positions of SNR candidates overlaid on the Digitized Sky Survey (DSS) image of the M 74 galaxy. 


\subsection{Spectral data}

To resolve the $[\mathrm{S}$ II] $\lambda \lambda 6716,6731$ lines and to separate [NII] $\lambda \lambda 6548,6583$ lines from $\mathrm{H} \alpha$, providing more accurate [S II]/H $\alpha$ values than imaging ratio values, we carried out spectral follow-up observations. The spectral data of our SNR candidates were obtained by SCORPIO (The Spectral Camera with the Optical Reducer for Photometrical and Interferometrical Observations), which was mounted on the $6 \mathrm{~m}$ BTA with a $2048 \times 2048$ pixel CCD (Afanasiev \& Moiseev 2005). A 3500$7200 \AA$ spectral coverage at $10 \AA$ resolution and a $1^{\prime \prime}$ wide slit were used during the observations.

The basic data reductions, flux and wavelength calibrations, and interstellar extinction correction were performed using IDL codes and IRAF (Image Reduction and Analyses Facility) packages (http: //iraf.noao.edu/docs/ docmain.html). Spectrophotometric standard stars from the Oke (1974) and Stone (1977) catalogs were also observed each night. We derived fluxes from the spectrophotometric standards and used these to calibrate the fluxes for spectral lines in our SNR spectra. Biases, internal lamp flats, and Ne-Ar calibration lamp frames were obtained for each observation set. The splot IRAF routine was used to measure the emission line fluxes of [S II] and $\mathrm{H} \alpha$. While obtaining spectral data for SNR candidates, a bright star was placed on the slit along with the target object. Galactic extinction correction was applied to the SNR spectra as part of the spectral analysis (Cardelli et al. 1989).

\section{Results and discussion}

\subsection{Results from optical imaging analysis}

We used the technique described by Blair \& Long (1997) to identify the SNR candidates. The identification technique was constructed through comparison of continuum-subtracted [S II] $\lambda \lambda 6716,6731$ and $\mathrm{H} \alpha$ images. Eventually, emission nebulae with region-integrated values of $[\mathrm{S} \mathrm{II}] / \mathrm{H} \alpha \geq 0.4$ were identified as the SNR candidates. A preliminary search to find these candidates were carried out by comparing the continuum subtracted [S II] and the $\mathrm{H} \alpha$ subfield images by a blinking technique. In the process, a full-field image of the galaxy was divided into regions of about $2^{\prime}$ by $2^{\prime}$ squares for the visual inspection and assessment of the fields for the candidates.

When a point-like source satisfied the criterion $[\mathrm{S} \mathrm{II}] / \mathrm{H} \alpha \geq$ 0.4 , we then marked it as an SNR candidate. After the assessment of all $2^{\prime}$ by $2^{\prime}$ subfields of M74, continuum-subtracted images were used to determine the ratio of $[\mathrm{S} \mathrm{II}] / \mathrm{H} \alpha$ counts. During this process, background counts were subtracted from the $[\mathrm{S} \mathrm{II}] / \mathrm{H} \alpha$ ratio images using annuli that were farther away, but centered on the selected candidates. The background extraction areas were also normalized to the source extraction areas. At the assumed distance of $7.3 \mathrm{Mpc}$ for M 74, our aperture size corresponds to $\sim 50 \mathrm{pc}$. During our interference filter imaging observations, seeing was $1.4^{\prime \prime}$, thus we cannot extract any radius information for our SNR candidates since it was limited by the seeing for the night.

We found nine emission nebulae consistent with the [S II] $/ \mathrm{H} \alpha \geq 0.4$ criterion for SNR identification with the technique described above. The list of new SNR candidates with corrected flux ratios are given in Table 2. The H $\alpha$ fluxes for SNR2, SNR3, and SNR5 were taken from spectral measurements, while the remaining ones were from image data. A limiting flux sensitivity of $\sim 10^{-15} \mathrm{erg} \mathrm{cm}^{-2} \mathrm{~s}^{-1}$ was calculated by choosing a structure with a limiting magnitude in the field of the galaxy. M 74 covers
Table 2. New optical SNR candidates detected in M74 in our observations.

\begin{tabular}{ccccc}
\hline \hline SNR Name & $\begin{array}{c}\text { RA } \\
(\mathrm{J} 2000.0)\end{array}$ & $\begin{array}{c}\text { Dec } \\
(\mathrm{J} 2000.0)\end{array}$ & {$[\mathrm{S} \mathrm{II}] / \mathrm{H} \alpha$} & $\begin{array}{c}I(\mathrm{H} \alpha) \\
\left(\mathrm{erg} \mathrm{cm}^{-2} \mathrm{~s}^{-1}\right)\end{array}$ \\
\hline SNR1 & $01: 36: 43.8$ & $+15: 49: 12.7$ & 0.47 & $1.5 \mathrm{E}-14$ \\
SNR2 & $01: 36: 31.5$ & $+15: 48: 50.3$ & 0.46 & $6.0 \mathrm{E}-15$ \\
SNR3 & $01: 36: 46.9$ & $+15: 45: 15.2$ & 0.91 & $2.9 \mathrm{E}-15$ \\
SNR4 & $01: 36: 45.3$ & $+15: 43: 06.6$ & 0.49 & $1.4 \mathrm{E}-14$ \\
SNR5 & $01: 36: 48.9$ & $+15: 43: 34.4$ & 0.58 & $2.8 \mathrm{E}-15$ \\
SNR6 & $01: 36: 45.1$ & $+15: 45: 25.3$ & 0.47 & $1.5 \mathrm{E}-14$ \\
SNR7 & $01: 36: 45.9$ & $+15: 44: 46.1$ & 0.45 & $1.6 \mathrm{E}-14$ \\
SNR8 & $01: 36: 45.9$ & $+15: 44: 27.1$ & 0.46 & $1.6 \mathrm{E}-14$ \\
SNR9 & $01: 36: 42.4$ & $+15: 44: 16.3$ & 0.40 & $1.7 \mathrm{E}-14$ \\
\hline
\end{tabular}

a total area of about $10.5 \times 9$ '.5 in the sky. Since the FOV of our CCD is 9'. $1 \times 9$ ' 1 , each filter image covered almost the full face of M 74. Therefore only a single observation for each filter was required. We zoomed on the full continuum-subtracted $\mathrm{H} \alpha$ image of M 74, and extracted four subfields, each of size 3.5 $\times 2.5$, and marked our SNR candidates on these images as displayed in Fig. 2.

\subsection{The follow-up optical spectroscopy}

We also conducted spectral observations to confirm the optical SNR candidates detected by optical imaging analysis. Among the nine candidates detected in M 74, we were only able to confirm three of them (SNR2, SNR3, and SNR5) with the derived specific line ratios [S II] $/ \mathrm{H} \alpha$ of $0.46,0.91$, and 0.58 , respectively. Their optical spectra are shown in Figs. 3-5. Observed line intensities relative to $\mathrm{H} \beta$, the $E_{(B-V)}$ values, and $\mathrm{H} \alpha$ intensities for the spectra of these three SNRs are given in Table 3. The rest of the six SNR candidates (SNR1, SNR4, SNR6, SNR7, SNR8, SNR9) could not be confirmed using the spectroscopic line ratio values. Several factors could be affecting the spectral [S II]/H $\alpha$ line ratio values. For instance, the long slit used for light acquisition may not always be accurately pointed on the objects. In such cases, contamination from nearby or overlapping H II emission regions could be affecting the line ratios. This seems likely for SNR1, SNR6, SNR7, SNR8, and SNR9, which were quite close to extensive H II emission regions as shown in Fig. 6. The spatial coordinates and sizes of these H II regions were taken from the catalog of Fathi et al. (2007) (this catalog can also be reached at http: //cdsweb.u-strasbg.fr/cgi-bin/qcat? $\mathrm{J} / \mathrm{A}+\mathrm{A} / 466 / 905 /)$. We thought that there could be significant $\mathrm{H}$ II emission contamination in these sources, as foreseen by Matonick \& Fesen (1997) and Blair \& Long (2004). They discuss some of their SNR candidates in relation to close-by H II regions (e.g., their SNR5 in NGC 6946, SNR39 in M 101, and SNR19 in M 81) with the angular separations of the SNRs being up to $15^{\prime \prime}-40^{\prime \prime}$ from H II regions. The angular separations of our candidates SNR1, SNR6, SNR7, SNR8, and SNR9 from the nearby $\mathrm{H}$ II regions calculated using our images were found in the range $5^{\prime \prime}$ to $10^{\prime \prime}$ in M 74. This corresponds to approximately the same distance scale when the relative distances of target galaxies are considered. On the other hand, we estimate that SNR1, SNR4, SNR8, and SNR9 have significant total [O I] emissions reaching about from $20 \%$ to $60 \%$ of the $\mathrm{H} \beta$ intensity. This is considered to be a secondary shock-heated gas indicator. In turn, a detectable [O I] $\lambda 6300$ emission is considered to be a viable argument for SNR identifications, in general (Blair \& Long 2004). 
(a)

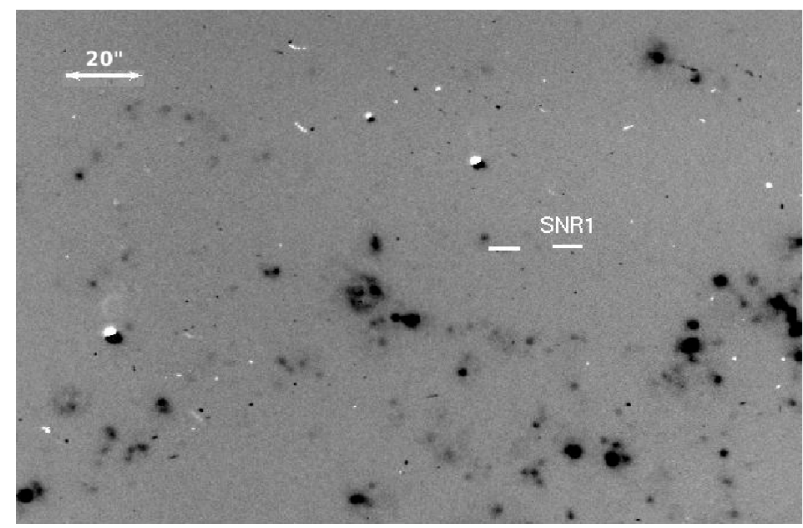

(c)

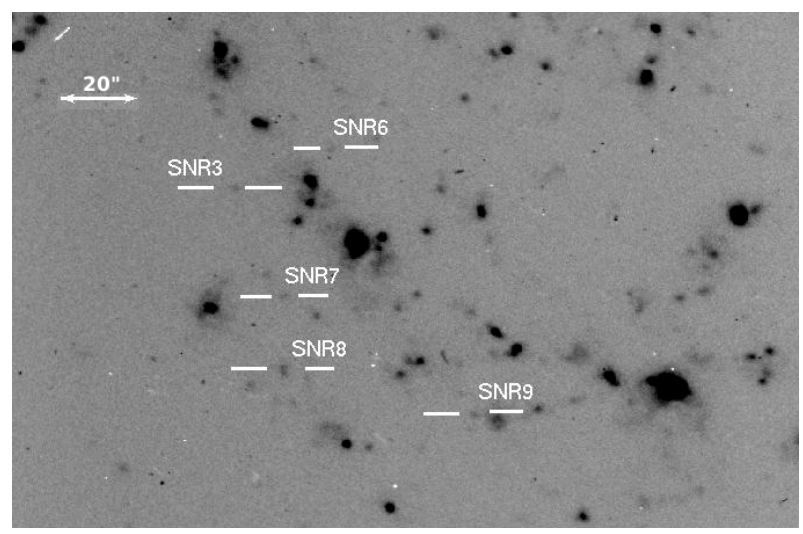

(b)

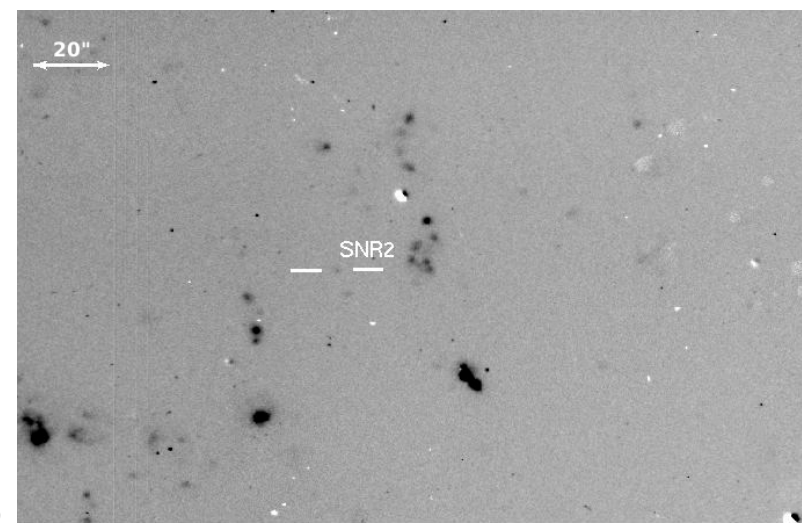

(d)

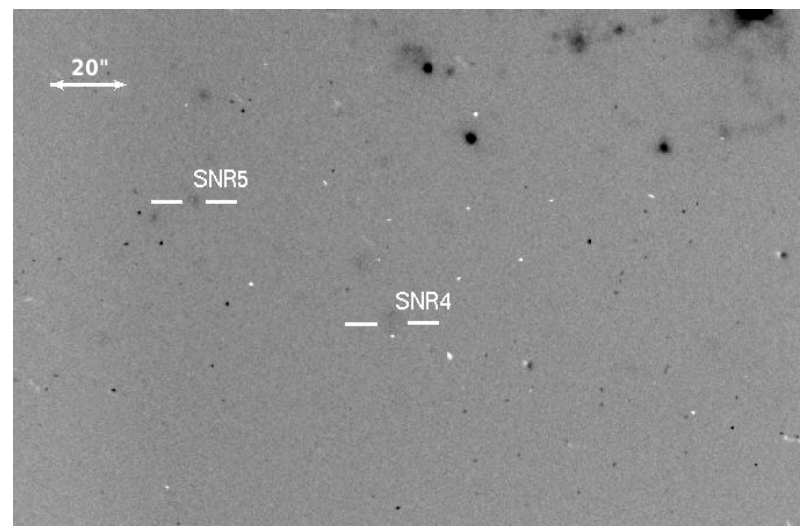

Fig. 2. Positions of SNR1 a), SNR2 b), SNR3, SNR6, SNR7, SNR8, SNR9 c), and SNR4, SNR5 d) overlaid on a 3.5 $\times 2.5$ subfield of continuumsubtracted $\mathrm{H} \alpha$ images of M 74 .

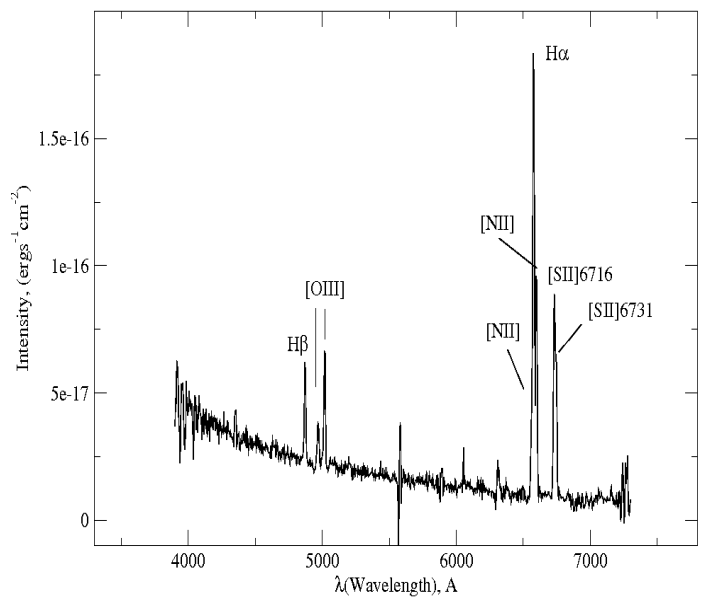

Fig. 3. Optical spectrum of SNR3 in M 74 obtained with BTA. Identified lines are indicated.

The detected SNR distribution in M 74 mostly appears in the southern field of the galaxy. Similar asymmetric distributions are observed in some other spiral galaxies, such as NGC 2403 and NGC 2903 (Matonick et al. 1997; Sonbas et al. 2009). Presently, the leading explanation for such asymmetries is that less SNRs are detected in the half of a given galaxy where $\mathrm{H}$ II regions are brighter. This also seems to be the case for M 74. We believe that this is not an artifact of our imaging or SNR identification techniques. We propose that, such asymmetries need to be discussed further with more galaxy examples and observations.

At the most basic level, average electron density in a nebula is measured through effects of collisional de-excitation. This

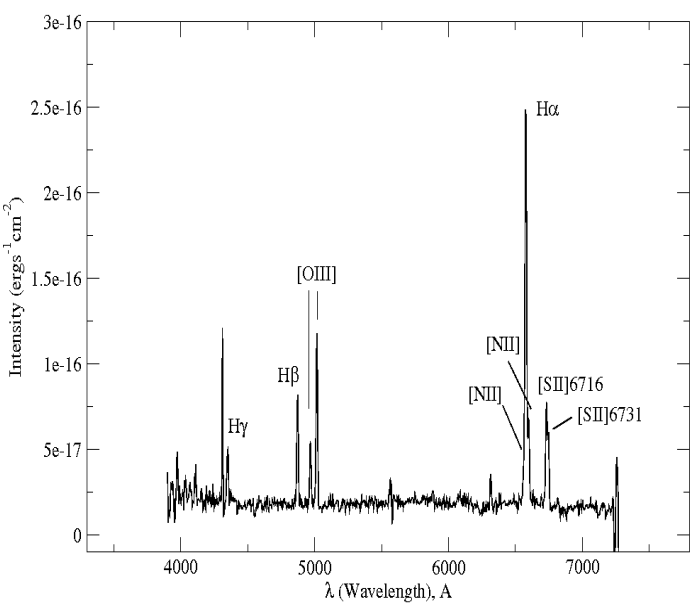

Fig. 4. Optical spectrum of SNR5 in M 74 obtained with BTA. Identified lines are indicated. (The strong spike just blueward of $\mathrm{H} \gamma$ is most probably caused by an unsubtracted cosmic hit on the CCD chip in use.)

is often done by comparing intensities of two lines from the same ion, emitted by different levels with the same excitation energy. One of the best examples of this procedure makes use of the electron density ratios [S II] $\lambda 6716 /[$ S II] $] 6731$ and $[\mathrm{OII}] \lambda 3729 /[\mathrm{OII}] \lambda 3726$. In our work we used the $[\mathrm{S} \mathrm{II}] \lambda 6716 /[\mathrm{S}$ II] $\lambda 6731$ line ratio from SNR spectra to calculate the electron density, $N_{\mathrm{e}}$. The Space Telescope Science Data Analysis System (STSDAS) task nebular.temden, based on the five-level atom approximation, calculates the required electron density given the electron temperature. Our calculated $N_{\mathrm{e}}$ value is $190 \pm 100 \mathrm{~cm}^{-3}$ for SNR3 corresponding to the line ratio of 1.24 for an assumed 
Table 3. Relative line intensities and observational parameters for SNRs spectroscopically observed in M 74.

\begin{tabular}{cccc}
\hline \hline Line & SNR2 & SNR3 & SNR5 \\
\hline $\mathrm{H} \beta(\lambda 4861)$ & 100 & 100 & 100 \\
$\mathrm{OIII}(\lambda 4959)$ & 3 & 60 & 50 \\
$\mathrm{OIII}(\lambda 5007)$ & 27 & 122 & 160 \\
$\mathrm{NII}(\lambda 5200)$ & - & 3 & - \\
$\mathrm{He}(\lambda 5876)$ & 13 & 33 & 9 \\
$\mathrm{OI}(\lambda 6300)$ & 17 & 21 & 32 \\
$\mathrm{OI}(\lambda 6364)$ & 14 & - & 7 \\
$\mathrm{NII}(\lambda 6548)$ & 30 & 154 & 56 \\
$\mathrm{H} \alpha(\lambda 6563)$ & 288 & 589 & 335 \\
$\mathrm{NII}(\lambda 6583)$ & 48 & 281 & 85 \\
$\mathrm{SII}(\lambda 6716)$ & 92 & 297 & 722 \\
$\mathrm{SII}(\lambda 6731)$ & 41 & 239 & 0.06 \\
\hline$E_{(B-V)}$ & 0.07 & 0.06 & 0.58 \\
$\mathrm{I}(\mathrm{H} \alpha)$ & $6.0 \mathrm{E}-15 \mathrm{erg} \mathrm{cm}-2 \mathrm{~s}^{-1}$ & $2.9 \mathrm{E}-15 \mathrm{erg} \mathrm{cm}^{-2} \mathrm{~s}^{-1}$ & $2.8 \mathrm{E}-15 \mathrm{erg} \mathrm{cm}^{-2} \mathrm{~s}^{-1}$ \\
{$[\mathrm{SII}] / \mathrm{H} \alpha$} & 0.46 & 0.91 & 0 \\
\hline
\end{tabular}

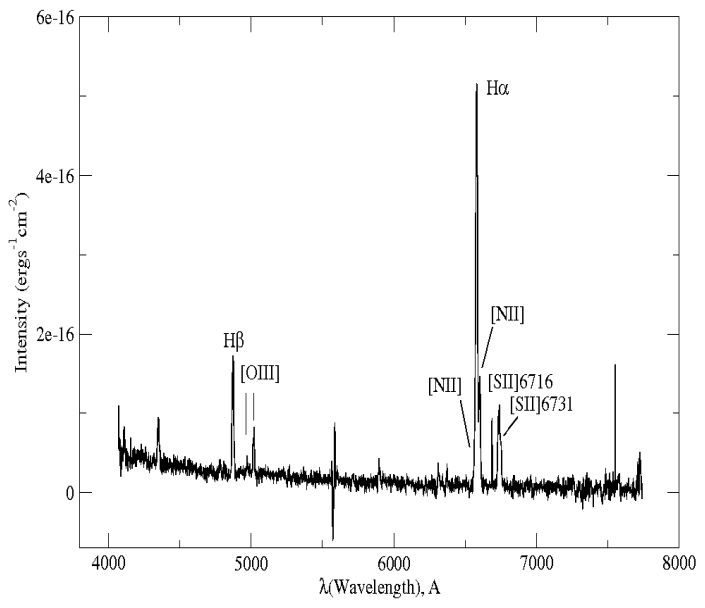

Fig. 5. Optical spectrum of SNR2 in M 74 obtained with BTA. Identified lines are indicated.

electron temperature of $T=10^{4} \mathrm{~K}$. The reason for the error term quoted here arises from the theoretical model losing its sensitivity for electron densities $<100 \mathrm{~cm}^{-3}$, which is in line with our spectral measurement sensitivities. With the same reasoning, although the line ratio values of $[\mathrm{S}$ II] $] \lambda 6716 /[\mathrm{S}$ II $] \lambda 6731>$ 1.46 are considered to correspond to a low density limit with $N_{\mathrm{e}} \leq 10 \mathrm{~cm}^{-3}$ (Osterbrock 1989), we can only quote an electron density for both SNR2 and SNR5 of simply $<100 \mathrm{~cm}^{-3}$. Using the shock abundance models by Dopita et al. (1984), the elemental abundances can be determined from the SNR spectra. Their models assume a shock velocity around or less than $100 \mathrm{~km} \mathrm{~s}^{-1}$ which is considered a low shock velocity. The same authors also make use of the fact that the behavior of [OIII] line is the most sensitive indicator of shock velocity variations. These variations fall rapidly for values less than $85 \mathrm{~km} \mathrm{~s}^{-1}$. In our case, $[\mathrm{OIII}] \lambda 5007 / \mathrm{H} \beta$ emission line ratios for SNR2, SNR3, and SNR5 were found as $0.27,1.22$, and 1.6 , respectively, corresponding to nebular propagation velocities of $<85 \mathrm{~km} \mathrm{~s}^{-1}$ (see Fig. 5 of Dopita et al. 1984). There are many SNRs that have low shock velocity values in a number of nearby galaxies (Matonick \& Fesen 1997; Blair \& Long 2004).

Among the working methods for separating SNRs from H II regions are the plots of $[\mathrm{S} \mathrm{II}] / \mathrm{H} \alpha$ versus $\mathrm{H} \beta$ flux and $[\mathrm{S} \mathrm{II}] / \mathrm{H} \alpha$ versus $[\mathrm{NII}] / \mathrm{H} \alpha$ line ratios. It was noted that many SNR candidates in a number of nearby galaxies show enhanced [NII] and very weak $\mathrm{H} \beta$ emissions when compared with the $\mathrm{H}$ II regions.

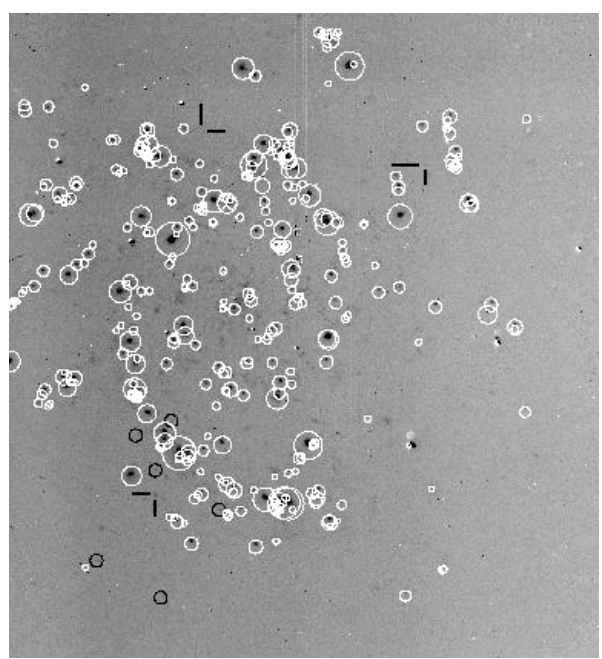

Fig. 6. H II regions and detected SNRs of M74. Perpendicular short lines indicate SNR1, SNR2, and SNR3, which are associated with Chandra X-ray point sources CXOUJ013631.7+154848, CXOUJ013644.0+154908, CXOUJ013646.0+154422, respectively. White circles indicate H II regions, and black circles indicate our six other SNRs.

We adopted the method by Blair \& Long (2004) based on their Fig. 18 where the same two plots for SNRs in M 83 are given. When we inserted our ratio values for detected SNRs into these plots (Fig. 7), we find similar tendencies. Our new SNR candidates are also located at the lower end, as would be expected. The observed low $\mathrm{H} \beta$ flux values are indicative of faint ionized hydrogen regions.

\subsection{Comparison with X-ray observations}

In a cross-check with the X-ray point source list of M 74, three X-ray sources (CXOUJ013631.7+154848, CXOUJ013644.0+154908, CXOUJ013646.0+154422, Soria et al. 2004) are found to be coincident with our optically detected SNRs (SNR1, SNR2, SNR8) taking $8^{\prime \prime}$ positional error circle around the objects. The error in association of SNR1 and SNR8 is $\sim 5^{\prime \prime}$ and SNR2 is $\sim 3^{\prime \prime}$. In Table 4 we list the optical and $\mathrm{X}$-ray associations for these SNRs. Among these, only SNR2 is a spectrally confirmed candidate. We used an archival Chandra pointed observation of M74 obtained in June 19, 2001 (with 
Table 4. Spatial associations between optically identified SNRs and Chandra-detected X-ray sources (from the combined 2001 June-October observation).

\begin{tabular}{cccc}
\hline \hline Optically detected SNR & RA & Dec & X-ray source \\
\hline SNR1 & $01: 36: 43.8$ & $+15: 49: 12.7$ & CXOUJ013644.0+154908 \\
SNR2 & $01: 36: 31.5$ & $+15: 48: 50.3$ & CXOUJ013631.7+154848 \\
SNR8 & $01: 36: 45.9$ & $+15: 44: 27.1$ & CXOUJ013646.0+154422 \\
\hline
\end{tabular}
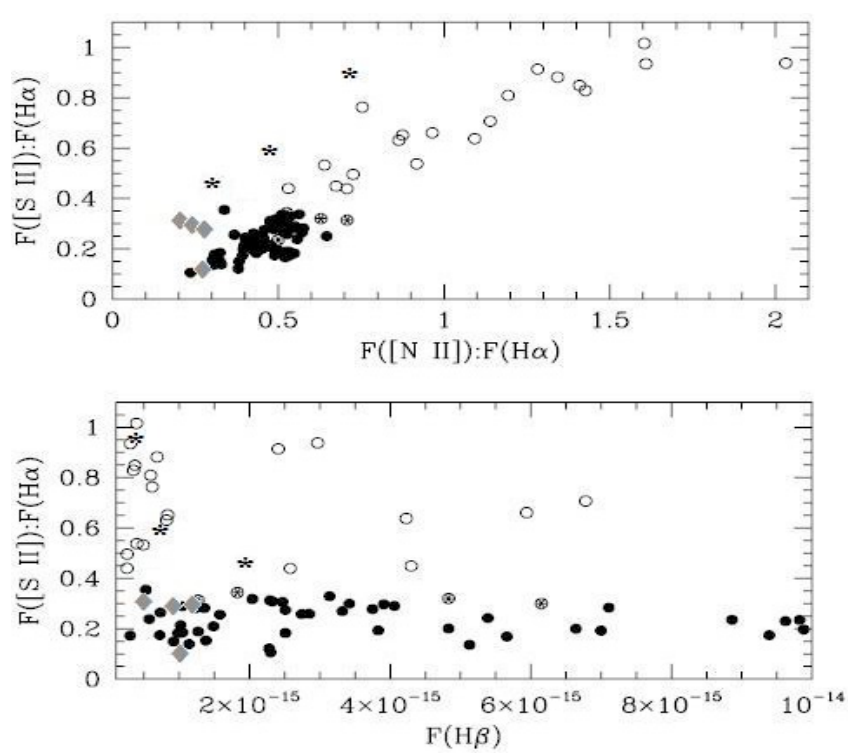

Fig. 7. [S II] $] / \mathrm{H} \alpha$ vs. [NII]/H $\alpha$ ratios and $[\mathrm{S} \mathrm{II}] / \mathrm{H} \alpha$ vs. $\mathrm{H} \beta$ fluxes. Stars indicate our spectroscopically identified SNRs, while diamonds the unidentified ones. These are compared with SNRs of M 83 Blair \& Long (2004) where open circles refer to spectroscopically identified SNRs, semifilled circles refer to spectroscopically unidentified SNRs, and filled circles refer to H II regions. Similar tendencies between SNRs of M 83 and M 74 are noticeable.

an exposure of $46.3 \mathrm{ks}$ ) to derive a spectrum of the SNR. We performed standard point source spectral analysis on the data and extracted source photons from the location of SNR2 with a circular extraction region of 12.5 , and background photons were extracted from a region normalized to the source photon extraction region. The full analysis was performed using CIAO 3.4 (http: //asc.harvard. edu/ciao3.4/index.html) and XSPEC 12.5.0ac software (Arnaud 1996).

We measured a count rate of $(1.5 \pm 0.5) \times 10^{-3} \mathrm{cts} \mathrm{s}^{-1}$ for SNR2. We derived a source spectrum and fitted this spectrum using the XSPEC model PSHOCK, a non-equilibrium ionization plasma emission model for shock-heated plasma appropriate for SNRs (Borkowski et al. 1996). The final Chandra spectrum of SNR2 is displayed in Fig. 8. The resulting spectral parameters are $N_{\mathrm{H}}=1.1_{>}^{+0.4} \times 10^{22} \mathrm{~cm}^{-2}, k T_{\text {plasma }}=10.8_{-9.4}^{+26.0} \mathrm{keV}$, the ionization timescale $\tau=n_{0} t=1.6_{>}^{+9.5} \times 10^{10} \mathrm{~s} \mathrm{~cm}^{-3}$, and the model normalization $K=1.1_{>}^{+2.8} \times 10^{-5} \mathrm{~cm}^{-5}$; the lower limit of the normalization is unconstrained. The $\chi_{v}^{2}$ of the fit is 1.3. The unabsorbed source flux is about $1.7 \times 10^{-13} \mathrm{erg} \mathrm{cm}^{-2} \mathrm{~s}^{-1}$, which translates to an X-ray luminosity $\sim 1.1 \times 10^{39} \mathrm{erg} \mathrm{s}^{-1}$ at the source distance of $7.3 \mathrm{Mpc}$. The derived plasma temperature yields a shock speed of $3100 \mathrm{~km} \mathrm{~s}^{-1}\left(k T_{\mathrm{s}}=(3 / 16) \mu m_{\mathrm{H}}\left(v_{\mathrm{s}}\right)^{2}\right)$ using Rankine-Huguenot jump conditions. The model normalization can be used to calculate the electron density of the shocked material as $n_{\mathrm{e}}=0.45 \mathrm{~cm}^{-3}$ (filling factor $f=1$ ) given a volume limited by the seeing, which corresponds to $50 \mathrm{pc}$ in size at the source distance. This value is consistent with the density derived

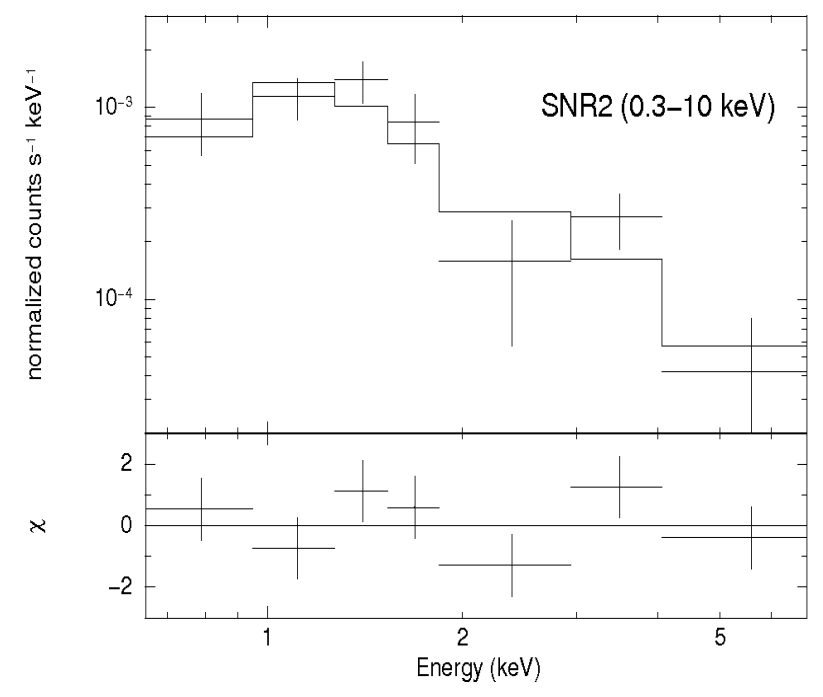

Fig. 8. X-ray spectrum of SNR2 obtained using archival Chandra data in 0.3-10.0 keV fitted with a neutral hydrogen absorption model, WABS and a PSHOCK non-equilibrium ionization plasma emission model (WABS*PHSOCK). The crosses indicate the data, the solid line is the fitted model (upper panel), and the residuals between the data and the model in standard deviations (lower panel).

from our optical line ratio analysis $n_{\mathrm{e}}<10 \mathrm{~cm}^{-3}$. Assuming that the ambient density $\mathrm{n}_{0}$ is not going to be more than the electron density, the ionization timescale yields a minimum lifetime of about 1120 years for SNR2. This indicates that SNR2 may be in the Sedov phase of its evolution.

It is important to note that the calculated luminosity for SNR2 is high for an SNR in the Sedov phase of evolution, and the calculated expansion speed cannot be supported with the blue shifts derived from the optical spectroscopy. Given this result, we also suspect that SNR2 may be a ULX (e.g. XMMU J013636.5+155036; Soria \& Kong 2002). We modeled the spectrum of SNR2 with a power law yielding an $N_{\mathrm{H}}=$ $2.6_{>}^{+0.5} \times 10^{21} \mathrm{~cm}^{-2}$, a $\Gamma=1.8_{-0.9}^{+1.5} \mathrm{keV}$, and a normalization $K_{\mathrm{pl}}=3.7_{>}^{+0.9} \times 10^{-6}$ with a $\chi_{v}^{2}=1.2$. Adding a blackbody component along with the power-law gives a $k T \sim 0.6-2 \mathrm{keV}$ and steepens the power law index to 1.8-5.4. A single blackbody fit gives a large $\chi_{v}^{2}$ above 1.5 .

Given the high shock plasma temperature that yields expansion speed that cannot be supported with the optical data and that the X-ray luminosity is on the order of $10^{39} \mathrm{erg} \mathrm{s}^{-1}$, we do not favor an old SNR interpretation for SNR2 as expected from the optical data analysis. We favor a ULX interpretation associated with an SNR. We expect that the very slow (less than $85 \mathrm{~km} \mathrm{~s}^{-1}$ ) expansion speed from the interpretation of the optical data will not yield detectable X-ray emission at the distance of M74. Then, any emission resulting from the point source remnant of the SN explosion will dominate the X-ray emission, though the SNR may dominate the optical emission. The ULX is expected to rejuvenate and ionize the nebulosity around it. Associations between SNRs and ULXs have been found or suggested before 
by Roberts et al. (2003), Abolmasov et al. (2007), Di Stefano \& Kong (2004), and Di Stefano et al. (2010).

We also checked the other two sources, SNR1 and SNR8. These sources were detected with $(1.0 \pm 0.5) \times 10^{-3}$ cts s$^{-1}$ and $(2.7 \pm 0.5) \times 10^{-3}$ cts s$^{-1}$, respectively. The fits with the PSHOCK model of the two sources result in a neutral hydrogen absorption of about $0.9 \times 10^{22} \mathrm{~cm}^{-2}$ and a $k T_{\text {plasma }}$ of about $34-73 \mathrm{keV}$ with an ionization timescale $\tau$ in the range $2-5 \times 10^{10} \mathrm{~s} \mathrm{~cm}^{-3}$. The spectral parameters yield an unabsorbed X-ray flux of $5 \times 10^{-15} \mathrm{erg} \mathrm{cm}^{-2} \mathrm{~s}^{-1}$ and $1 \times 10^{-13} \mathrm{erg} \mathrm{cm}^{-2} \mathrm{~s}^{-1}$ for SNR1 and SNR8, respectively, translating to an X-ray luminosity of $\sim 5 \times 10^{37} \mathrm{erg} \mathrm{s}^{-1}$ and $\sim 7 \times 10^{38} \mathrm{erg} \mathrm{s}^{-1}$. Given these spectral parameters and assuming these are SNRs we can speculate that the expansion speed is 2-3 times faster than SNR2 for these two SNRs. The electron density is about 1.4 to 2.3 times less than SNR2, and the minimum lifetime is about 1570-2600 years for SNR1 and SNR8 compared with SNR2. In general, one expects that the SNR shock decelerates in time, so that these longer lifetimes contradict the faster shock speeds and high X-ray temperatures detected for these two SNRs in comparison with SNR2. These shock temperatures and expansion speeds are not supported by our optical data. We note that these two sources are not confirmed SNRs with our ratio measurements using optical spectroscopy. Thus, we suspect they are SNRs and favor an $\mathrm{X}$-ray binary or an AGN interpretation for the two sources. For the same three sources XMM-Newton data yielded confirming spectra. Therefore, no details are repeated here.

Finally, we calculated an X-ray flux limit of $<9.0 \times$ $10^{-15} \mathrm{erg} \mathrm{cm}^{-2} \mathrm{~s}^{-1}$ with a luminosity limit of $<5.0 \times 10^{37} \mathrm{erg} \mathrm{s}^{-1}$ for the rest of the SNRs in our list, including the two other spectrally confirmed sources SNR3 and SNR5, since we find no positional coincidence with any of the source listings of M74.

\section{Conclusions}

We conducted a survey of SNRs using optical imaging and spectroscopic measurements in the nearby spiral galaxy M 74. In this survey, we used blinking between continuum-subtracted $\mathrm{H} \alpha$ and continuum-subtracted [S II] images to deduce SNR candidates for a given ratio between the lines. The SNRs were confirmed by additional spectroscopic observations and, finally, by comparison of the optically detected SNRs with archived Chandra observations. Nine SNRs were identified in M 74 by our method. Three of these have shown positional coincidences with X-ray sources. SNR2 is our best candidate to be an SNR using optical wavelength analysis, and the X-ray source is consistent with a ULX interpretation, making it one of the few associations between an SNR and a ULX. We calculated an X-ray flux upper limit of $9.0 \times 10^{-15} \mathrm{erg} \mathrm{cm}^{-2} \mathrm{~s}^{-1}$ and a luminosity upper limit of $5.0 \times 10^{37} \mathrm{erg} \mathrm{s}^{-1}$ for our new SNR candidates excluding SNR1, SNR2, and SNR8. Our spectroscopically detected SNRs have shock velocities $<85 \mathrm{~km} \mathrm{~s}^{-1}$, indicating that they fall into a low shock-velocity range thereby imply very old remnants from which little X-ray emission is expected.

According to the SN rate calculation of Matonick \& Fesen (1997) in such galaxies, about half of all SNe are of type Ib/c or type II, produced by massive stars. In turn, only half of these $\mathrm{SNe}$ are located in regions with enough ambient density to produce a detectable SNR. This means that only about a quarter of all SN events may leave easily detectable optical remnants. In this respect, four times more $\mathrm{SN}$ events should have exploded in M 74, and we detected nine SNRs. With this reasoning, we would expect to see $\sim 40$ SNRs. If the optically observable lifetime of an SNR is assumed to be 20000 years (Braun et al. 1989), a crude SN occurrence rate for M 74 can be estimated giving a value of about 1 per $\sim 500$ years. In the light of the observations of two recent $\mathrm{SNe}$ from $\mathrm{M} 74$, our result can be reconciled if we accept that more $\mathrm{SNe}$ occur at places that are difficult to reach optically, (i.e. in gas-rich regions of the galaxy).

Acknowledgements. We thank the TUBITAK National Observatory (TUG) and the Special Astrophysical Observatory (SAO) for their support for observing times and equipment.

\section{References}

Abolmasov, P. K., Fabrika, S. N., \& Sholukhova, O. N. 2007, Black Holes from Stars to Galaxies-Across the Range of Masses (Cambridge University Press), Proc. IAU Symp., 238, 229

Afanasiev, V. L., \& Moiseev, A. V. 2005, Astron. Lett., 31, 194

Arnaud, K. A. 1996, Astronomical Data Analysis Software and Systems V, ed. G. H. Jacoby, \& J. Barnes, ASP Conf. Ser., 101, 17

Auld, R., Minchin, R. F., Davies, J. I., et al. 2006, 371, 1617

Blair, W. P., \& Long, K. S. 1997, ApJS, 108, 261

Blair, W. P., \& Long, K. S. 2004, ApJS, 155, 101

Borkowski, K., Szymkowiak, A. E., Blondin, J. M, \& Sarazin, C. L. 1996, ApJ, 466, 866

Braun, R., \& Walterbos, R. A. M. 1993, A\&AS, 98, 327

Braun, R., Goss, W. M., \& Lyne, A. G. 1989, ApJ, 340, 355

Cardelli, J. A., Clayton, G. C., \& Mathis, J. S. 1989, ApJ, 345, 245

Cornett, R. H., O’Connell, R. W., Greason, M. R., et al. 1994, 426, 553

Di Stefano, R., \& Kong, A. K. H. 2004, ApJ, 609, 710

Di Stefano, R., Primini, F. A., Liu, J., Kong, A., \& Patel, B. 2010, AN, 331, 205

D’Odorico, S., Dopita, M. A., \& Benveuti, P. 1980, A\&AS, 40, 67

Dopita, M. A., Binette, L., Dodorico, S., \& Benvenuti, P. 1984, ApJ, 276, 653

Elmegreen, B. G., Elmegreen, D. M., Chandar, R., Whitmore, B., \& Regan, M. 2006, ApJ, 644, 879

Fathi, K., Beckman, J. E., Zurita, A., et al. 2007, A\&A, 466, 905

Ghavamian, P., Blair, W. P., Long, K. S., et al. 2005, AJ, 130, 539

Gordon, S. M., Kirshner, R. P., Long, K. S., et al. 1998, ApJS, 117, 89

Gordon, S. M., Duric, N., Kirshner, R. P., Goss, W. M., \& Viallefond, F. 1999, ApJS, 120, 247

Hendry, M. A., Smartt, S. J., Maund, J. R., et al. 2005, MNRAS, 359, 906

Hyman, S. D., Calle, D., Weiler, K. W., et al. 2001, ApJ, 551, 702

Jacoby, G. H., Africano, J. L., \& Quigley, R. J. 1987, ASP Publications, 99, 672 Krauss, M. I., et al. 2003, ApJ, 630, 228

Lacey, C. K., \& Duric, N. 2001, ApJ, 560, 719

Lacey, C., Duric, N., \& Goss, W. M. 1997, ApJS, 109, 417

Leievre, M., \& Roy, J. R. 2000, ApJ, 120, 1306

Magnier, E. A., Prins, S., van Paradijs, J., et al. 1995, A\&A, 114, 215

Mathewson, D. S., \& Clarke, J. N. 1973, ApJ, 180, 725

Matonick, D. M., \& Fesen, R. A. 1997, ApJS, 112, 49, 107

Matonick, D. M., Fesen, R. A., Blair, W. P., \& Long, K. S. 1997, ApJS, 113, 333 Monet, D., et al. 1998, CDS/ADC Collection of Electronic Catalogues, 1252

Oke, J. B. 1974, ApJS, 27, 21

Osterbrock, D. E. 1989, S\&T, 78, 491

Pannuti, T. G., Duric, N., Lacey, C., et al. 2000, ApJ, 544, 780

Pannuti, T. G., Swartz, D. A., Duric, N., \& Urosevic, D. 2002, American Astronomical Society, 200, 3505

Pannuti, T. G, Schlegel, E. M. \& Lacey, C. K. 2007, AJ, 133, 1361

Pence, W. D., Snowden, S. L., Mukai, K., \& Kuntz, K. D. 2001, ApJ, 561, 189 Roberts, T. P., Goad, M. R., Ward, M. J., \& Warwick, R. S. 2003, MNRAS, 342 , 709

Sharina, M. E., Karachentsev, I. D., \& Tikhonov, N. A. 1996, A\&AS, 119, 499

Sonbas, E., Akyuz. A., \& Balman, S. 2009, A\&A, 493, 1061

Stone, R. P. S. 1977, ApJ, 218, 767

Soria, R., \& Kong, A. K. H. 2002, ApJ, 572, 33

Soria, R., Pian, E., \& Mazzali, P. A. 2004, A\&A, 413, 107

van Dyk, S. D., Li, W., \& Filippenko, A. V. 2003, PASP, 115, 1289 